\title{
THE FUNDAMENTAL THEOREM ON TORSION CLASSES OF LATTICE-ORDERED GROUPS \\ BY
}

JORGE MARTINEZ

\begin{abstract}
This paper generalizes the earlier notion of a torsion class to a setting where its significance can be fully realized. The dual notion of a torsion-free class is herein defined and the fundamental Connection Theorem is proved. In addition, a few restrictions are considered, in particular, how to view the application of the main theorem to the hereditary classes.
\end{abstract}

I. Introduction. This author introduced the concept of a torsion class in [6]. Since then the notion has been examined by several people.

Here we extend the definition as follows. All classes are assumed to be closed under $l$-isomorphy. Unless we indicate otherwise all groups are assumed to be in multiplicative notation.

A class $\mathcal{T}$ is a torsion class if it is closed with respect to $l$-homomorphic images, and, in addition, for each family of convex $l$-subgroups $\left\{C_{i} \mid i \in I\right\}$ of the $l$-group $G$ such that each $C_{i} \in \mathcal{T}$, we also have $C=\bigvee_{i \in I} C_{i} \in \mathcal{T}$. If $\mathcal{T}$ is a torsion class and $G$ is an $l$-group, let $\mathcal{T}(G)$ stand for the join of all the convex $l$-subgroups of $G$ belonging to $\mathcal{T}$. It is evident that $\mathscr{T}(G)$ is characteristic, i.e. invariant under all the $l$-automorphisms of $G$. In particular, $\mathcal{T}(G)$ is an $l$-ideal.

LEMMA 1. Suppose $\mathcal{T}$ is a torsion class. We then have

(1) If $C$ is a convex l-subgroup of $G$ then $\mathscr{T}(C) \leqslant \mathcal{T}(G)$.

(2) If $\phi: G \rightarrow H$ is an l-homomorphism then $[\mathcal{T}(G)] \phi<\mathcal{T}(H)$.

(3) $\mathscr{T}(\mathcal{T}(G))=\mathscr{T}(G)$.

Conversely, if we associate to each l-group $G$ a convex l-subgroup $\mathscr{D}(G)$ subject to (1), (2) and (3) above, and let $\mathcal{T}=\{G \mid \mathscr{D}(G)=G\}$, then $\mathcal{T}$ is a torsion class and $\mathscr{T}(G)=\mathscr{Q}(G)$ for each l-group $G$.

The proof of Lemma 1 is straightforward. It proceeds in the same vein as that of Proposition 1.1 of [6]. The proof may be found in [7]. In fact, most of the material in this paper occurs in [7], which was written as a series of lecture notes. In this article we shall omit most routine or highly technical arguments-such as the proof of Lemma 1-and refer the reader to [7] and also [6]. [7] should be viewed as a supplement.

Received by the editors May 9, 1979.

AMS (MOS) subject classifications (1970). Primary 06A15, 06F15; Secondary 08C99.

${ }^{1}$ The author gratefully acknowledges the support of the National Science Foundation (Grant \# MCS78-02036). 
We have thus discarded the 'other' defining condition for torsion classes. If $\mathcal{T}$ is a torsion class and is also closed with respect to taking convex $l$-subgroups, we shall henceforth say that $\mathcal{T}$ is a hereditary torsion class.

Lemma 1 assures us that we may view a torsion class from the standpoint of its associated radical $\mathcal{T}(G)$. A torsion class $\mathcal{T}$ is hereditary if and only if $\mathcal{T}(C)=C \cap$ $\mathcal{T}(G)$ for each $l$-group $G$ and each convex $l$-subgroup $C$ in $G$.

If $\mathcal{T}$ is any torsion class, let $\hat{\mathcal{T}}=\{G \mid \mathcal{T}(G)=1\}$. It is fairly easy to show that $\hat{\mathcal{T}}$ is a class closed under

(i) taking convex $l$-subgroups, and

(ii) subdirect products (meaning that if $\phi: G \rightarrow \Pi_{i \in I} G_{i}$ is an l-embedding such that each 'projection' $\phi_{i}: G \rightarrow G_{i}$ is onto, and each $G_{i} \in \hat{\mathcal{T}}$, then $G \in \hat{\mathfrak{T}}$ ).

Moreover, $\hat{\mathcal{J}}$ is closed under extensions: suppose $G$ is an $l$-ideal of $H$ and both $G$ and $H / G$ belong to $\hat{\mathcal{T}}$. Then $\mathcal{T}(H) \leqslant G$ by (2) of Lemma 1 . By (1) and (3) of that lemma $\mathcal{T}(H)=\mathcal{T}(\mathcal{T}(H)) \leqslant \mathcal{T}(G)=1$. Hence $H \in \hat{\mathcal{T}}$.

Any class $\mathscr{X}$ closed under (i) and (ii) above will be called a torsion-free class.

If $\mathcal{X}$ is a torsion-free class and $G$ is an l-group, let $\mathcal{X}(G)$ be the intersection of all the $l$-ideals $A$ of $G$ for which $G / A \in \mathfrak{X}$. Clearly, $\mathscr{X}(G)$ is the smallest $l$-ideal with this property. Once again it is evident that $\mathscr{X}(G)$ is characteristic.

We now have the dual to Lemma 1; the proof can be found in [7, Proposition 2.2.2].

LeMma 2. Suppose $\mathcal{X}$ is a torsion-free class.

(a) $\mathfrak{X}(A) \leqslant \mathscr{X}(G)$, for each convex l-subgroup $A$ of $G$.

(b) If $K$ is an l-ideal of $G$ and $\mathscr{X}(G / K)=1$, then $K>\mathscr{X}(G)$.

(c) $\mathfrak{X}(G / \mathcal{X}(G))=1$.

Conversely, suppose we associate to each l-group $G$ an l-ideal $\mathscr{G}(G)$ subject to (a), (b) and (c) above. If $\mathcal{X}=\{G \mid \mathcal{G}(G)=1\}$, then $\mathcal{X}$ is a torsion-free class and $\mathscr{X}(G)=$ $g(G)$ for each l-group $G$.

Thus, as for torsion classes, we may view torsion-free classes two ways: as classes of $l$-groups or as functions which take on values in the lattice of convex $l$-subgroups of an l-group. We call $\mathscr{X}(G)$ the $\mathcal{X}$-torsion-free radical of $G$, or simply the $\chi$-radical if the context is clear.

Before proceeding to the main theorem, let us give some examples. We shall not reproduce the list from [6]; the reader should know that [7] has an extensive review of those examples. We should however single out Holland's theorem (from [3]) stating that each equational class of $l$-groups is a torsion class. It is also worth mentioning that no proof is known of the claim (made in [6]) that the class of vector lattices is a torsion class.

From a different point of view, each equational class is a torsion-free class. In fact, the equational classes are the only ones which are both torsion and torsionfree. An equational class $\mathfrak{V}$ then has two radicals associated with it: its torsion radical $\mathcal{V}_{t}$ and its torsion-free radical $\mho_{q}$, which is the usual verbal subgroup, as in Neumann [8], for groups. In $\S 6$ of [7] we look at some relationships between $\mathfrak{V}_{t}$ and $\mathfrak{V}_{q}$. Those results have no place in this article. 
The passage from $\mathcal{T}$ to $\hat{\mathcal{T}}$ (where $\hat{\mathcal{T}}=\{G \mid \mathcal{T}(G)=1\}$ ) yields a torsion-free class if one starts with a torsion class $\mathcal{T}$. We can also go back: let $\mathcal{X}$ be a torsion-free class, and $\hat{X}=\{G \mid \mathcal{X}(G)=G\}$. $\hat{X}$ is the class of l-groups having no nontrivial $l$-homomorphic images in $\mathscr{X}$. Evidently, $\hat{X}$ is closed under $l$-homomorphic images. It is also straightforward to check that $\hat{X}$ is closed under joins of convex l-subgroups. Thus, $\hat{\mathfrak{X}}$ is a torsion class.

Notice that here too $\hat{X}$ is closed under extensions: if $A$ is an $l$-ideal of $G$ and both $A$ and $G / A$ are in $\hat{X}$, let $K$ be an $l$-ideal of $G$ such that $G / K \in \mathcal{X}$. Then $A \cap K$ is an $l$-ideal of $A$ and $A / A \cap K \simeq A K / K$ which is a convex $l$-subgroup of $G / K$. Hence $A / A \cap K \in \mathfrak{X}$, which implies that $A=A \cap K$, or $A<K$. But $G / K \simeq(G / A) /(K / A)$ and so $K=G$. Hence $G \in \hat{\mathcal{X}}$.

If $\mathcal{T}$ is a torsion class, $\hat{\mathfrak{T}}$ is called the opposite torsion-free class; if $\mathcal{X}$ is a torsion-free class, we refer to $\hat{X}$ as the opposite torsion class.

For example, if $\mathcal{X}$ is the class of subdirect products of real groups, then $\hat{X}$ is the class of $l$-groups having no convex $l$-subgroups which are both maximal and normal. This class is not hereditary! There exist archimedean l-groups having no maximal convex $l$-subgroups-a suitable $D(X)$ will do-while each principal convex $l$-subgroup is a subdirect product of real groups.

Some other examples of nonhereditary torsion classes:

A. Let $\mathcal{T}$ be the class of $l$-groups $G$ in which each positive element lies beneath a finite-valued element. (To review the definition of finite-valuedness see [2] or [7].) The opposite torsion-free class is the class of $l$-groups having no special elements.

B. Let $\mathcal{T}$ be the class of $l$-groups $G$ in which each positive element lies beneath a normal-valued element (again, see [2] or [7] for a definition of this notion). The opposite class consists of those $l$-groups without normal-valued elements.

C. If $\alpha$ is an infinite cardinal, take $\mathcal{V}_{\alpha}$, the class of l-groups in which each element exceeds one having at most $\alpha$ values. The opposite class: every element must have more than $\alpha$ values.

II. The main theorem. We are now ready for the Connection Theorem between torsion and torsion-free classes. If $\mathcal{T}$ (resp. $\mathcal{X}$ ) is a torsion (resp. torsion-free) class, let $\mathcal{J}^{*}=(\hat{\mathfrak{T}})^{\wedge}\left(\right.$ resp. $\left.\mathcal{X}^{*}=(\hat{\mathcal{X}})^{\wedge}\right)$.

Connection Theorem. The functions $\mathcal{T} \rightarrow \hat{\mathcal{T}}$ and $\mathcal{X} \rightarrow \hat{\mathscr{X}}$ between torsion and torsion-free classes form a Galois connection. In addition, $\mathcal{T}_{(G)}<\hat{\mathcal{T}}(G)=\mathcal{T}^{*}(G)$ for each l-group $G$ and each torsion class $\mathcal{T}$, while $\mathscr{X}(G) \geqslant \hat{X}(G)=\mathcal{X}(G)$ for each torsion-free class $\mathfrak{X}$.

Proof. It is evident that the maps $\mathscr{T} \rightarrow \hat{\mathcal{T}}$ and $\mathcal{X} \rightarrow \hat{X}$ are order-inverting. Further, if $G \in \mathcal{T}$, it can have no $l$-homomorphic images in $\hat{\mathcal{J}}$ except 1 ; hence $G \in \mathcal{T}^{*}$. Put differently, $\mathcal{T}(G) \leqslant \mathcal{T}^{*}(G)$ for all $l$-groups $G$. Since $\mathcal{T}^{*}$ is closed with respect to extensions: $\mathcal{T}\left(G / \mathcal{J}^{*}(G)\right)=1$, so that $G / \mathcal{T}^{*}(G) \in \hat{\mathcal{J}}$. Furthermore, if $K$ is an $l$-ideal of $G$ so that $G / K \in \hat{\mathcal{T}}$, then $\mathcal{T}^{*}(G) K / K$ is an $l$-homomorphic image of $\mathcal{T}^{*}(G)$ belonging to $\hat{\mathcal{T}}$. This implies that $\mathcal{T}^{*}(G) K=K$ : that is, $\mathcal{T}^{*}(G)<K$. By Lemma $2, \mathscr{T}^{*}(G)=\hat{\mathfrak{T}}(G)$ for each l-group. 
The proof that $\mathscr{X}(G) \geqslant \hat{X}(G)=\mathscr{X} *(G)$ for all torsion-free classes is analogous. We shall omit it.

From this it is clear that $\hat{\mathcal{T}}=\left(\mathcal{T}^{*}\right)^{\wedge}$ and $\hat{X}=\left(X^{*}\right)^{\wedge}$, for all $\mathcal{T}$ and $\mathcal{X}$, and therefore that the maps in question form a Galois connection.

In the context of any Galois connection the iteration of the maps involved (such as $\mathcal{T} \rightarrow \mathcal{T}^{*}$ and $\mathfrak{X} \rightarrow \mathfrak{X}^{*}$, in our setting) define closure operations. (For a discussion of this idea, the reader is referred to Birkhoff [1].) Let us examine the 'closed' objects in this situation.

We say that a class $C$ of $l$-groups is complete if it is closed under extensions. Then the following comes out of our main theorem.

THEOREM 3. A torsion class $\mathcal{T}$ is complete if and only if $\mathcal{T}=\mathcal{T}^{*}$. Moreover, if $\mathscr{U}$ is any torsion class then $\mathcal{U}^{*}$ is the smallest complete torsion class containing $\mathscr{U}$.

A dual statement holds for torsion-free classes.

Proof. We need only prove the second statement, and we already know that $\mathcal{U}^{*}$ is a complete torsion class. Now suppose $\mathcal{T}$ is a complete torsion class containing थ. Then $\hat{\mathcal{T}} \leqslant \hat{U}$; this implies that $G / \mathcal{T}(G) \in \hat{\mathrm{U}}$. (Note. The completeness of $\mathcal{T}$ guarantees that $G / \mathcal{T}(G) \in \hat{\mathcal{T}}$.) So if $G \in \mathcal{U}^{*}$ then $G / \mathcal{T}(G)$ is an $l$-homomorphic image of $G$ belonging to $\hat{U}$; conclusion: $G=\mathcal{T}(G)$, and $\mathcal{U}^{*}<\mathcal{T}$.

We are justified then in calling $\mathcal{T}^{*}$ the completion of $\mathcal{T}$, and likewise $\mathcal{X}^{*}$ the completion of a torsion-free class $\mathcal{X}$. Next, we wish to outline an alternate approach to these completions; it involves transfinite induction. Let us handle torsion classes first. In the sequel the letters $\alpha, \beta, \ldots$ will be used for ordinal numbers. $G$ is an $l$-group and $\mathcal{T}$ is an arbitrary torsion class.

Let $\mathfrak{T}^{1}=\mathcal{T}$. Assume that $\mathcal{T}^{\alpha}$ have been defined for all ordinals $\alpha<\beta$. If $\beta$ is a limit ordinal let $\mathcal{T}^{\beta}(G)=\cup_{\alpha<\beta} \mathcal{T}^{\alpha}(G)$. If on the other hand $\beta$ has a predecessor $\beta-1$, we define $\mathcal{T}^{\beta}(G)$ by the equation

$$
\left[\mathcal{T}^{\beta}(G)\right] /\left[\mathcal{T}^{\beta-1}(G)\right]=\mathscr{T}\left(G / \mathcal{T}^{\beta-1}(G)\right) \text {. }
$$

We leave it to the reader to verify that in both instances $\mathcal{T}^{\beta}$ is a torsion class (assuming that each $\mathscr{T}^{\alpha}$ is, for $\alpha<\beta$ ).

Next, define $\mathcal{T}^{\text {tim }}=\cup \mathcal{T}^{\alpha}$, over all ordinals. It turns out, after a routine verification, that $\mathfrak{J}^{\text {lim }}=\mathcal{T}^{*}$.

With torsion-free classes the situation is as follows: for any torsion-free class $\mathscr{X}$, let $\mathfrak{X}^{1}=\mathfrak{X}$. Then assume $\mathfrak{X}^{\alpha}$ has been defined for each ordinal $\alpha<\beta$. If $\beta$ is a limit ordinal, let $\mathfrak{X}^{\beta}(G)=\cup_{\alpha<\beta} \mathfrak{X}^{\alpha}(G)$. Otherwise, define $\mathfrak{X}^{\beta}(G)=$ $\mathcal{X}\left(\mathcal{X}^{\beta-1}(G)\right)$, where $\beta-1$ is $\beta$ 's predecessor. Again, there are certain details to be verified, all strictly routine.

As with torsion classes, define $\mathscr{X}^{\text {lim }}=\cup \mathfrak{X}^{\alpha}$ (over all the ordinals). Then $\mathfrak{X}^{\lim }=\mathfrak{X}^{*}$.

We summarize.

Theorem 4. Suppose $\mathcal{T}$ and $\mathcal{X}$ are torsion and torsion-free classes, respectively. Suppose $G$ is an l-group. We obtain a sequence of torsion classes $\mathscr{T}^{1}<\mathscr{T}^{2}<\ldots<$ $\mathcal{T}^{\alpha} \leqslant \cdots$, where $\mathcal{T}^{1}=\mathcal{T}$ and 


$$
\mathscr{T}^{\beta}(G)\left\{\begin{array}{l}
=\cup_{\alpha<\beta} \mathscr{T}^{\alpha}(G), \quad \text { if } \beta \text { is a limit ordinal, } \\
\text { defined as }\left[\mathcal{T}^{\beta}(G)\right] /\left[\mathscr{T}^{\beta-1}(G)\right]=\mathscr{T}\left(G / \mathcal{T}^{\beta-1}(G)\right), \text { otherwise. }
\end{array}\right.
$$

Then $\mathcal{T}^{*}=\cup \mathcal{T}^{\alpha}$.

We also get a sequence of torsion-free classes: $\mathfrak{X}^{1} \leqslant \mathscr{X}^{2} \leqslant \cdots \leqslant \mathfrak{X}^{\alpha}<\cdots$, where $\mathfrak{X}^{1}=\mathfrak{X}$ and

$$
\mathfrak{X}^{\beta}(G)= \begin{cases}\cup_{\alpha<\beta} \mathfrak{X}^{\alpha}(G), & \text { if } \beta \text { is a limit ordinal, } \\ \mathcal{X}\left(\mathfrak{X}^{\beta-1}(G)\right), & \text { otherwise. }\end{cases}
$$

Again $X^{*}=\cup \mathscr{X}^{\alpha}$.

III. Hereditary classes. To begin we ask the following question: if $\mathcal{T}$ is a hereditary torsion class what special properties does its opposite class have? Recall that $\mathcal{T}$ is hereditary if and only if $\mathcal{T}(A)=A \cap \mathcal{T}(G)$ for each l-group $G$ and each convex $l$-subgroup $A$ of $G$. It is also known that if $\mathcal{T}$ is hereditary then so is its completion $\mathcal{T}^{*}$ (Theorem 1.6, from [6]).

Furthermore, if $\mathcal{T}$ is hereditary and $\mathcal{X}=\hat{\mathfrak{g}}$ then $\mathscr{X}$ satisfies

(H1) $\mathfrak{X}(A)=\mathscr{X}(G) \cap A$ for each convex $l$-subgroup $A$ of $G$.

(H2) If $\left\{A_{i} \mid i \in I\right\}$ is a family of convex $l$-subgroups of $G$, and $A=\bigvee A_{i}$, then if each $A_{i} \in \mathfrak{X}, A \in \mathfrak{X}$.

(H3) If $A$ is a dense convex $l$-subgroup of $G$ and $A \in \mathscr{X}$ then $G \in \mathcal{X}$. ( $A$ is dense in $G$ if each $1<g \in G$ exceeds a positive element $a \neq 1$ in $A$.)

(H4) If $P$ is a normal polar subgroup of $G$ and $G \in \mathcal{X}$ then $G / P \in \mathcal{X}$.

(Note. $P$ is the polar of the convex $l$-subgroup $A$ if it is the largest convex $l$-subgroup for which $P \cap A=1$. We then write $P=A^{\prime}$. A polar subgroup then is one for which $P=P^{\prime \prime}$. We observe that $A$ is dense in $G$ if $G=A^{\prime \prime}$. For more details on polars we refer the reader to Conrad [2].)

Now then, we know that $(\mathrm{H} 1)$ holds because $\mathcal{X}(G)=\mathcal{T}^{*}(G)$, by the Connection Theorem. (H1) clearly implies (H3). Presently we shall prove that (H3) always implies (H4). It is just as evident that (H1) implies (H2).

First we prove that any torsion-free class $\mathcal{X}$ satisfying $(\mathrm{H} 3)$ is complete.

LEMMA 5. If $\mathcal{X}$ is a torsion-free class satisfying (H3) then $\mathcal{X}$ is complete.

Proof. Suppose $A \leqslant G$ is an $l$-ideal such that $A$ and $G / A$ both belong to $\mathcal{X}$. By Lemma $2, A \geqslant \mathfrak{X}(G)$ and therefore $\mathfrak{X}(G) \in \mathscr{X}$. In addition, $\mathfrak{X}(G)^{\prime}$ is $l$-isomorphic to an $l$-ideal of $G / \mathcal{X}(G)$, which implies that $\mathscr{X}(G)^{\prime} \in \mathscr{X}$. Next, since $\mathscr{X}(G) \mathcal{X}(G)^{\prime}$ is $l$-isomorphic to the direct product of $\mathscr{X}(G)$ and $\mathcal{X}(G)^{\prime}$ it is in $\mathcal{X}$. Now, invoking (H3), since $\mathscr{X}(G) \mathcal{X}(G)^{\prime}$ is dense in $G, G \in \mathcal{X}$.

Then, in particular, if $\mathscr{X}$ satisfies $(\mathrm{H} 1)$ it is complete.

Proposition 6. (H3) implies (H4).

Proof. Suppose $\mathcal{X}$ is a torsion-free class satisfying (H3). If $P$ is a normal polar of the $l$-group $G$ which belongs to $\mathscr{X}$, then $P^{\prime} \in \mathcal{X}$. In addition, $P^{\prime} \simeq P^{\prime} P / P$, which is dense in $G / P$. Hence $G / P \in \mathcal{X}$.

From Lemma 5 we have the following characterization. 
Proposition 7. Suppose $\mathcal{X}$ is a torsion-free class. Then $\mathcal{X}$ satisfies (H1) if and only if $\mathfrak{X}$ is complete and $\hat{X}$ is hereditary.

Proof. Lemma 5 and the Connection Theorem.

We still need to say a word or two about the independence of conditions (H1) through (H4). We already have the implications:

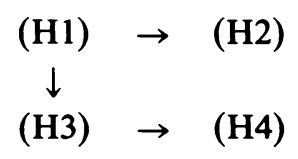

Consider also the following ' $l$-ideal' versions of (H1) and (H3):

(HLl) $\mathfrak{X}(A)=\mathscr{X}(G) \cap A$ for each $l$-ideal $A$ of $G$.

(HL3) If $A$ is a dense $l$-ideal of $G$ and $A \in \mathcal{X}$, then $G \in \mathcal{X}$. It is proved in [7] that (HL1) and (HL3) are equivalent. The theorem (2.5.3) goes like this.

THEOREM 8. For a torsion-free class $\mathscr{X}$ the following are equivalent.

(1) $\hat{X}$ is closed under taking l-ideals and $\mathscr{X}$ is complete.

(2) $\mathscr{X}$ satisfies (HL1).

(3) $\mathcal{X}$ satisfies (HL3).

Clearly (H1) $\rightarrow$ (HL1) and (H3) $\rightarrow$ (HL3). In addition, the proof of Proposition 6 applies to show that (HL3) $\rightarrow(\mathrm{H} 4)$. In view of Theorem 8 we would like to know whether $(\mathrm{H} 3) \rightarrow(\mathrm{H} 1)$. Now recall: equational classes of $l$-groups are the only ones which are both torsion and torsion-free classes. So any noncomplete equational class satisfies (H2) and not (H3) or (HL3). (It is easy to see that (HL3) also implies completeness.) The class of subdirect products of real groups satisfies (H4)-see Martinez [5]-but neither (H3) nor (H2). The class of archimedean groups possesses (H4) and (H2)-for the latter see Kenny [4]-but not (H3).

What follows is an example to show that (H2) does not imply (H4). Consider the torsion-free class $\mathcal{X}$ of $l$-groups having no finite-valued elements. Suppose $G$ is an $l$-group and $\left\{A_{i} \mid i \in I\right\}$ is a family of convex $l$-subgroups of $G$. If $A=\bigvee A_{i}$ has a finite-valued element then it also has a special element. But if $a>1$ in $A$ is special and is written $a=a_{1} a_{2} \ldots a_{k}$ with $1<a_{t} \in A_{i_{i}}$, then at least one of the $a_{i}$ is special. Therefore, if each $A_{i}$ is in $\mathscr{X}$ then $A \in \mathfrak{X}$; thus, $X$ satisfies (H2).

On the other hand, suppose $A$ is an (additive) $l$-group without finite-valued elements. Let $P$ stand for the $l$-group of periodic real sequences. On $A \times P$ define $0<\left(a ; s_{1}, s_{2}, \ldots\right)$ if each $s_{n} \geqslant 0$, but if $s_{1}=0$ then $a>0$. It is not hard to see that $A \times P$ is an $l$-group without finite-valued elements. If $B=(A \times\{0\})^{\prime}=$ $\left\{\left(0 ; 0, s_{2}, \ldots\right) \mid s_{n}\right.$ real $\}$, then $(A \times P) / B$ is a direct lexicographic extension of $A$ by the reals, which does have finite-valued elements. Thus (H4) fails.

Finally, an example to show (HL1) does not imply (H1). The verification is not difficult; we have presented it in [7], and refer the reader to it. Let $g$ stand for the torsion class of all $l$-groups in which each $l$-ideal is a cardinal summand. (That is, $G \in \mathcal{G}$ if and only if $G=A^{\prime} A$ for each $l$-ideal $A$ of $G$.) $\mathcal{g}$ and $\mathcal{G}^{*}$ are closed under $l$-ideals and hence satisfy (HL1), but neither is hereditary! 
Our picture of implications between the various conditions mentioned here is as follows.

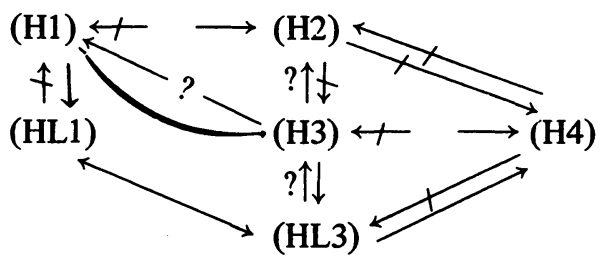

The question marks indicate the implications which are still undecided.

\section{REFERENCES}

1. G. Birkhoff, Lattice theory, Amer. Math. Soc. Colloq. Publ., vol. 25, Amer. Math. Soc., Providence, R. I., 1967.

2. P. Conrad, Lattice-ordered groups, Tulane University Lecture Notes, 1970.

3. W. C. Holland, Varieties of l-groups are torsion classes, Czechoslovak Math. J. (to appear).

4. O. Kenny, Dissertation, University of Kansas, 1975.

5. J. Martinez, Archimedean-like classes of lattice-ordered groups, Trans. Amer. Math. Soc. 186 (1973), 33-49.

6. __ Torsion theory for lattice-ordered groups, Czechoslovak Math. J. 25 (100) (1975), 284-299.

7. _ A general theory of torsion classes for lattice-ordered groups, University of Florida notes.

8. H. Neumann, Varieties of groups, Ergebnisse der Math. und ihrer Grenzgebiete, Band 37, Springer-Verlag, Berlin and New York, 1967.

Department of Mathematics, University of Florma, Gainesville, Florida 32611 